\title{
Measuring text readability with machine comprehension: a pilot study
}

\author{
Marc Benzahra ${ }^{1,2}$ and François Yvon ${ }^{1}$ \\ ${ }^{1}$ LIMSI, CNRS, Université Paris-Saclay, F-91405 Orsay, France \\ ${ }^{2}$ Glose, 53 rue du rocher, F-75008 Paris, France \\ \{marc.benzahra, francois.yvon\}alimsi.fr
}

\begin{abstract}
This article studies the relationship between text readability levels and automatic machine understanding systems. Our hypothesis is that the simpler a text is, the better it should be understood by a machine. We thus expect a strong correlation between readability levels on the one hand, and performance of automatic reading systems on the other hand. We test this hypothesis with several understanding systems based on language models of varying strengths, measuring this correlation on two corpora of journalistic texts. Our results suggest that this correlation is quite small and that existing comprehension systems are far to reproduce the gradual improvement of their performance on texts of decreasing complexity.
\end{abstract}

\section{Introduction}

The automatic evaluation of the readability of texts is an old subject (see, for example, (DuBay, 2007) for an historical account), which continues to arouse great interest from the Natural Language Processing (NLP) community; a recent analysis of the state-of-the-art is given by Collins-Thompson (2014). Checking that a written document is expressed in a language that is accessible to its target audience is essential in many situations: for instance to ensure that the information conveyed by the text is properly understood or to allow sufficient engagement of the reader in the reading activity (Dietrich, 2004). These issues are relevant both for the dissemination of general purpose information (e.g. news articles) and more targeted information such as drug leaflets, administrative texts or legal documents. They apply to various readerships: children in first or second language learning situations, adults with varying levels of education or with disabilities, etc.

The classical measures of readability are based on crude approximations of the syntactic complex- ity (using the average sentence length as a proxy) and lexical complexity (average length in characters or syllables of words in a sentence). One of the most well-known measure along these lines is the Flesch-Kincaid readability index (Kincaid et al., 1975), which combines these two measures into a global score. This approach has recently been renewed by the use of supervised statistical learning methods capable of integrating into the prediction of readability a very large number of linguistic characteristics (Schwarm and Ostendorf, 2005; Petersen and Ostendorf, 2009; Vajjala and Meurers, 2012; François and Fairon, 2012; Vajjala and Meurers, 2014; Brunato et al., 2018) aimed at capturing readability indices at the lexical, syntactic, semantic and even discursive levels. It can be argued that these enhanced feature sets are able to take into account so-called cognitive factors (Feng et al., 2009).

However, these approaches depend on the availability of texts annotated with their difficulty levels, which are often defined in relation to a particular task or readership. The elicitation of these annotations is a complex operation, which requires either the implementation of understanding measurement protocols on controlled populations, using for example cloze tests to evaluate understanding (Taylor, 1953; Oller Jr., 1973); or the work of highly qualified experts, at the risk of observing disagreements between annotators (Petersen and Ostendorf, 2009). They also require automatically extracting linguistic features from texts, which existing NLP tools only partially achieve, for a limited number of languages.

In this paper, we study an alternative method that could help assess the readability level of texts in an unsupervised manner. Our main hypothesis, developed in $\S 2$, is that automatic text understanding systems (machine reading) having made remarkable progress (Hermann et al., 2015; Dhin- 
gra et al., 2017; Yu et al., 2018), ${ }^{1}$ it might become possible to use them to assess the readability of texts. The preliminary question of interest here is therefore whether the ability of automatic comprehension systems to respond to gap-filling questions correlates with the difficulty of the text, and can be help to measure readability. To answer this question, we study several simplistic machine understanding systems, described in $\S 2.4$ and empirically examine the correlation between their performance and the actual complexity of texts, measured by humans (see $\S 3$ ). Our main findings are that when comprehension is evaluated using cloze tests, all the systems make very little difference, if any, between texts of varying complexities, suggesting that we should reconsider our evaluation scheme, or our set of comprehension systems, or both, to achieve a behavior that would be more similar to human's performance.

\section{Simulating human comprehension}

\subsection{The design of readability scores}

From a bird's eye view, the construction of measures of text readability involves two steps: (a) having human subjects perform controlled comprehension tests on texts of interest, which determine the empirical readability of the texts for the concerned population of readers (typically characterized by a level of education, or a level of language proficiency for second language learners); (b) measuring a set of indices in texts that effectively predict empirical readability values and learning the parameters of a function relating these surface cues with difficulty.

The approach we explore relies on the assumption that automatic comprehension systems having greatly improved, it should become possible to use them to simulate step (a) above, and thus to use the results of machine comprehension tests on a given text to directly evaluate its empirical readability, dispensing with the need to perform step (b).

As a first step in this direction, we need to check whether that performance of machine comprehension systems should exhibit a form of dependency to the actual readability of the text: the simpler the text, the better they should be understood by a

\footnotetext{
${ }^{1}$ See however the work of Jia and Liang (2017) or Kaushik and Lipton (2018), who claim that quantitative progresses, measured on standard question answering tasks, mainly reflect an improvement in the ability of these systems to perform surface matches between questions and answers.
}

machine. This is the main question that we empirically address below, using an automatic understanding architecture based on statistical Language Models (LMs), and evaluating the task of automatic comprehension using cloze tests.

A second important question will concern our ability to build comprehension systems that accurately simulate the understanding ability of a target population (of children, of language learners, of people with disabilities, etc). We delay the systematic study of this question for further work, but contend that our methodology is compatible with this objective, as it can handle, as will be seen, the design of comprehension systems of varying strengths.

To reiterate, our objective is to study the performance of comprehension systems as a possible measure of text readability: our final goal is not so much to improve comprehension systems but to see how they could be used as proxies of human comprehension.

\subsection{Testing machine comprehension}

The measurement of automatic comprehension of texts is an old and difficult issue. By analogy with measures of human understanding, two main methods are commonly used: comprehension questions (Richardson et al., 2013; Rajpurkar et al., 2016) and cloze tests.

The use of cloze tests to assess the performance of comprehension systems has for instance been proposed by Hill et al. (2016), which studies the ability of various neural models to fill in blanks in sentences from children's stories, introducing the CBT (Children Book Tests) corpus.

This technique has also been used in several previous studies (Bimbot et al., 2001; Zweig and Burges, 2012) to evaluate language models: the former reference establishes a direct link between perplexity and filling-the-gap tests; the latter introduces the Sentence Completion Challenge in which gaps and distractors are carefully selected.

One of the challenges of recent work on this issue is the development of realistic test sets, which can only be answered by a deep understanding of the text (Paperno et al., 2016; Xie et al., 2018), using for instance information regarding words partof-speech or syntactic role of the deleted word. 


\begin{tabular}{|c|c|c|}
\hline Cloze test & Prediction & Ground truth \\
\hline $\begin{array}{l}\text { Herring is a type of fish } \ldots \text {--- it swims closer to San Francisco } \\
\text { than before. Herring is a favorite food of sea }{ }_{----} \text {, so they might } \\
\text { have followed those fish to }{ }_{----} \text {. Sea lions might also like the } \\
\text { pier because ... }\end{array}$ & $\begin{array}{r}\text { otters } \\
\text { San }\end{array}$ & $\begin{array}{l}\text { lions } \\
\text { shore }\end{array}$ \\
\hline $\begin{array}{l}\text { They have twin daughters named Barbara and Jenna } \\
\text { family has a dog _---- } \text { Barney and a } \text { The }_{---} \text {named India. }\end{array}$ & $\begin{array}{r}\text { named } \\
\text { cat }\end{array}$ & $\begin{array}{r}\text { named } \\
\text { cat }\end{array}$ \\
\hline $\begin{array}{l}\text { Neil Armstrong was the first man to step onto the moon .___ put } \\
\text { an American flag up on the moon . } \\
\text { to }\end{array}$ & $\begin{array}{r}\text { He } \\
\text { Earth }\end{array}$ & $\begin{array}{r}\mathrm{He} \\
\text { He } \\
\text { Earth }\end{array}$ \\
\hline
\end{tabular}

Table 1: Cloze test examples filled with a GPT-2 language model.

\subsection{Cloze test generation, completion and evaluation}

We stick here to a much simpler form of cloze testing, based on a uniform random strategy to select the deleted words. We leave for future work the use of more sophisticated methods specifically designed to generate difficult tests.

In practice, each test document is automatically divided into $N$ passages of the same size; in each passage, $M$ positions are randomly selected uniformly and correspond to the words that will be blanked out. These words will have to be recovered by the comprehension system, which has access to the complete left context of the gap since the beginning of the passage. In our experiments, we use $N=5$ and $M=3$.

One interesting property of cloze tests is that they provide ways to analyze the complexity of gap fillings with respect to arbitrary linguistic lexical descriptors; they also provide ways to compute difficulty levels separately for each sentence and check for instance its dependency with respect to the length of the context. Table 1 shows examples of Cloze tests given to our language models.

Gap filling performance is measured as the average number of words that are correctly predicted in a text (noted p@1) in the tables below. This metric being very strict, we also report the number of times the correct word appears in the first 5,25 , and 50 candidates. Many other techniques could be entertained to weaken the p@1 metric, for example by considering the similarity (formal or distributional) between the predicted word and the reference. Again, we leave the study of these alternative metrics to future work.

\subsection{A comprehension architecture based on language models}

Our comprehension systems are neural language models based on recurrent structures (RNN) (Elman, 1990; Mikolov et al., 2010) with LSTM cells (Hochreiter and Schmidhuber, 1997), which continue to deliver (near) state-of-the-art results for statistical language modelling (Melis et al., 2018). Once trained, the prediction of a missing word is performed by only looking at its left context, ordering the candidate words $w$ at position $t$ according to $P\left(w_{t}=w \mid w_{<t}\right)$. $^{2}$

Among all the degrees of freedom of the method, we simply varied (a) the quality of models using the same architecture, and (b) the training data. We acknowledge that it would also be possible to use other architectures for language models (e.g. n-grams models (Chen and Goodman, 1999)); to use character-based models (Sutskever et al., 2011; Kim et al., 2016) or subword-based models that could accommodate open vocabularies; or to train more sophisticated RNN models (Yang et al., 2018).

It would likewise be possible to use more complex algorithms to predict missing words (e.g. by using left and right contexts), or even by testing text understanding systems that are more representative to the current state of-the-art (Dhingra et al., 2017; Yu et al., 2018).

As a first step in this direction, we also experiment with a self-attentional language-model (AlRfou et al., 2018; Liu et al., 2018) using the Transformer architecture (Vaswani et al., 2017), from

\footnotetext{
${ }^{2}$ In this respect, our use of LMs for readability continues the work of Collins-Thompson and Callan (2004); Petersen and Ostendorf (2009) and many others who used LM scores as a predictor of the complexity level.
} 


\begin{tabular}{|l||r|r|r|r|r|r|r|r|r|}
\hline \multicolumn{1}{|c||}{} & \multicolumn{3}{c|}{ WikiText-2 } & \multicolumn{3}{c|}{ WikiText-103 } & \multicolumn{3}{c|}{ Wiki-Simple } \\
\cline { 2 - 10 } & \#params & \#epochs & ppl & \#params & \#epochs & ppl & \#params & \#epochs & ppl \\
\hline RNN & $19 \mathrm{M}$ & 100 & 90 & $113 \mathrm{M}$ & 3 & 87 & $44 \mathrm{M}$ & 50 & 51 \\
\hline AWD & $19 \mathrm{M}$ & 100 & 78 & $113 \mathrm{M}$ & 3 & 137 & $44 \mathrm{M}$ & 50 & 65 \\
\hline
\end{tabular}

Table 2: Basic statistics regarding language models

the implementation of Radford et al. (2019). Such models are capable to effectively handle very long range dependencies and have been able to achieve good performance on some recent machine comprehension benchmarks.

\section{Experiments}

In this section, we compare the automatic comprehension measures produced by our language models with readability scores produced by humans, as well as with other automatic standard indices of readability. We start with details regarding the implementation of our language models.

\subsection{Language Model Training}

\subsubsection{Corpora}

To train our language models, we use two standard corpora of articles from the English Wikipedia: ${ }^{3}$ WikiText-2 and WikiText-103, that have already been used as benchmarks in language modeling tasks (Merity et al., 2017). As can be seen from the statistics in Table 3, these corpora mostly differ in their size and vocabulary.

We also use an additional set of articles from the English version of the simplified Wikipedia: ${ }^{4}$ Wiki-Simple. ${ }^{5}$ Following Merity et al. (2017), this "simple" dataset is pre-processed as follow: tokens with less than 3 occurrences are replaced by the "<unk $>$ " token as in WikiText-2 and WikiText-103.

\begin{tabular}{|l|r|r|r|}
\hline Source & Docs & Tokens & Types \\
\hline Wiki-2 & 720 & $2.5 \mathrm{M}$ & $33 \mathrm{~K}$ \\
\hline Wiki-103 & 28591 & $103 \mathrm{M}$ & $260 \mathrm{~K}$ \\
\hline Wiki-Simple & $60 \mathrm{~K}$ & $7.9 \mathrm{M}$ & $96 \mathrm{~K}$ \\
\hline
\end{tabular}

Table 3: Language model training corpora

All language modelling corpora in Table 3 are tokenized by splitting text for each space separator

\footnotetext{
${ }^{3}$ https://en.wikipedia.org

${ }^{4}$ https://simple.wikipedia.org/

${ }^{5}$ Version 2.0 http://www.cs.pomona.edu/ akauchak/simplification/
}

(") encountered. It is expected that training models on the Wiki-Simple corpora should yield poorer gap-filling systems than the other two corpora, as these systems will be exposed to simpler sentence structures and a more basic vocabulary.

\subsubsection{LM Implementation issues}

Our own RNN language models use the following architecture: 3 hidden layers, each containing 512 recurrent cells. Training starts with randomly initialized embeddings, and is performed using SGD (Robbins and Monro, 1951), dividing by 4 the learning rate (initialized to 30) every 5 epochs if the loss does not decrease. We use a drop-out of 0.1 for the embedding layer, 0.3 for the LSTM layers and 0.4 for the output layer ; the batch size is 64, and backpropagation context is 64. We borrow two additional techniques from Merity et al. (2018): the use of variable-length backpropagation sequences and the use of two regularization terms penalizing the parameters of the output layers: activation regularization and temporal activation regularization (see reference for details). The weight matrices at the output of LSTM after the linear projection and the word embedding are shared to avoid overfitting (Inan et al., 2017; Press and Wolf, 2017) and to reduce the number of parameters. This implementation is referred to as RNN below.

We contrast our implementation with that of Merity et al. (2018) ${ }^{6}$ trained with the same parameter values as our RNN implementation, random seed value is 1882 . This implementation is referred to as AWD below. Basic statistics regarding these models (size, test set perplexity) are in Table 2, suggesting that we were able to have a diverse set of models of varying strengths ${ }^{7}$.

Last, we also report results obtained with a selfattentional architecture, directly using the smaller version (context size is 768 and number of param-

\footnotetext{
${ }^{6}$ https: / / github.com/salesforce/ awd-lstm-lm

${ }^{7}$ Note that the perplexity score do not directly compare across training corpus, as the underlying event space is slightly different for each training corpus.
} 

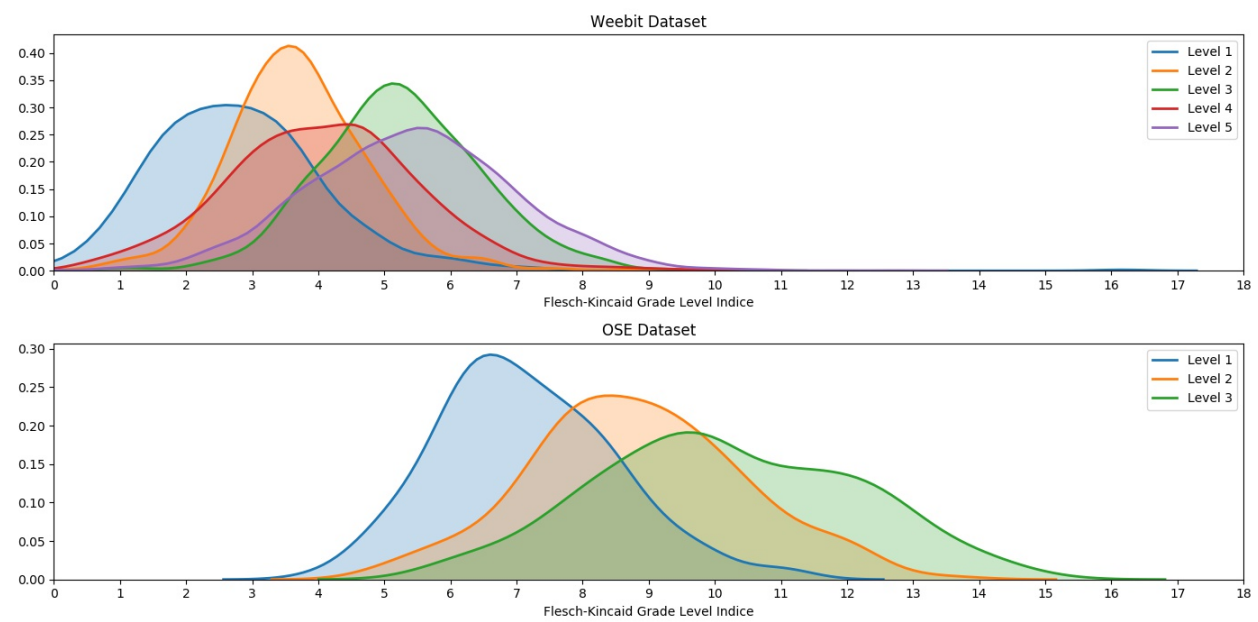

Figure 1: Distribution of the Flesch-Kincaid index by complexity level

eters $117 M$ against a context size of 1600 units and $1.5 B$ parameters for the largest and closed source version) of the trained models of Radford et al. $(2019)^{8}$, referred to as GPT-2 in the Tables below. This model uses a vocabulary of $50 \mathrm{~K}$ variable length subword units (Sennrich et al., 2016) and is trained on a corpus of $8 \mathrm{M}$ documents; according to its authors, it achieves a perplexity of respectively 29 and 37 on WikiText -2 and WikiText-103. As this model is based on sub-word units, it can not directly be used to fill gaps. Our greedy heuristic gap-filling procedure is the following: (a) first generate a list of $K$ most likely subword units; (b) greedily expend rightwards these $K$ hypotheses until one word is generated for at most $t$ time steps. We use $t=10$ in our experiments.

\subsection{Test data and evaluation}

The reference annotations used to validate our method mostly come from two sources: Weebit (Vajjala and Meurers, 2012) and OneStopEnglish (OSE for short) (Vajjala and Lucic, 2018). The first identifies 5 levels of complexity in educational news articles published online (on the WeeklyReader and BBC-Bitesize Web sites), covering learners aged from 7 to 16 . As a merger of two sources, the distribution of categories is quite unbalanced, with more texts for the fifth level than for all the other categories taken together.

\footnotetext{
${ }^{8}$ Taken with their associated API from https://github.com/huggingface/ pytorch-pretrained-BERT
}

The second test set $^{9}$ also contains extracts of journalistic texts originally from the newspaper The Guardian. Each document has been rewritten twice by experts to correspond to two less advanced reading levels, and thus distinguishes three levels of readability. Statistics on these test corpora are in Table 4.

\begin{tabular}{|l|r|r|r|r|}
\hline Source & Docs & Tokens & Gaps & Levels \\
\hline Weebit & 10486 & $3.9 \mathrm{M}$ & $157 \mathrm{~K}$ & 5 \\
\hline OSE & 567 & $450 \mathrm{~K}$ & $8.5 \mathrm{~K}$ & 3 \\
\hline
\end{tabular}

Table 4: Basic statistics of test corpora

Figure 1 displays the distribution of the FleschKincaid Grade Level (FKGL) for these two corpora. Complexity levels are distributed nearly as expected for the Weebit corpus apart from levels 3 and 4 where the latter seems simpler than foreseen, with large overlapping spans. It was already noted by (Vajjala and Meurers, 2012) that the actual readability level of each test was difficult to predict accurately based on the sole FKGL. OSE complexity levels are also in agreement with the Flesch-Kincaid index, and in agreement with the numbers reported in (Vajjala and Lucic, 2018); again we see a large overlap between levels for this index. Overall, OSE texts are somewhat more complex than Weebit's, with OSE level 1 comparable in difficulty to Weebit level 4.

Both sources were pre-processed with regular expressions to discard bits of text that were recurrent such as advertisement footers for companies (BBC, WeeklyReader, MetaMetrics) and material

\footnotetext{
${ }^{9}$ See https : / / zenodo.org/record/1219041.
} 


\begin{tabular}{|c|c|c|c|c|c|c|c|c|c|c|c|c|}
\hline \multirow[b]{2}{*}{ p@... } & \multicolumn{4}{|c|}{ WikiText-2 } & \multicolumn{4}{|c|}{ WikiText-103 } & \multicolumn{4}{|c|}{ Wiki-Simple } \\
\hline & 1 & 5 & 25 & 50 & 1 & 5 & 25 & 50 & 1 & 5 & 25 & 50 \\
\hline RNN (1) & 0.10 & 0.21 & 0.33 & 0.39 & 0.12 & 0.24 & 0.37 & 0.44 & 0.12 & 0.27 & 0.40 & 0.47 \\
\hline RNN (2) & 0.09 & 0.20 & 0.29 & 0.35 & 0.11 & 0.23 & 0.34 & 0.41 & 0.11 & 0.23 & 0.36 & 0.43 \\
\hline RNN (3) & 0.08 & 0.19 & 0.28 & 0.34 & 0.10 & 0.22 & 0.33 & 0.39 & 0.10 & 0.22 & 0.33 & 0.39 \\
\hline$\overline{\mathrm{RNN}}(\tau)$ & 0.05 & 0.06 & 0.11 & 0.12 & 0.06 & 0.06 & 0.09 & 0.10 & 0.11 & 0.13 & 0.17 & 0.19 \\
\hline AWD (1) & 0.11 & 0.22 & 0.34 & 0.40 & 0.12 & 0.23 & 0.35 & $\overline{0.42}$ & 0.12 & 0.25 & 0.36 & 0.43 \\
\hline AWD (2) & 0.10 & 0.20 & 0.31 & 0.37 & 0.11 & 0.21 & 0.32 & 0.37 & 0.11 & 0.22 & 0.32 & 0.38 \\
\hline AWD (3) & 0.09 & 0.19 & 0.30 & 0.35 & 0.10 & 0.21 & 0.31 & 0.37 & 0.10 & 0.21 & 0.30 & 0.36 \\
\hline$\overline{\mathrm{AWD}(\tau)}$ & 0.05 & $\overline{0.08}$ & 0.09 & 0.11 & 0.06 & 0.07 & 0.09 & 0.11 & $\overline{0.11}$ & 0.11 & 0.15 & 0.17 \\
\hline
\end{tabular}

Table 5: Completion rates broken down per readability level for gap filling systems of variable strength tested on the OSE dataset. Kendall tau-b correlation is reported as $\tau$.

\begin{tabular}{|l||r|r|r|r||r|r|r|r||r|r|r|r||}
\hline \multicolumn{1}{|c||}{} & \multicolumn{4}{|c||}{ Wiki $@$ Text-2 } & \multicolumn{4}{c||}{ WikiText-103 } & \multicolumn{4}{|c||}{ Wiki-Simple } \\
\cline { 2 - 15 } & 1 & 5 & 25 & 50 & 1 & 5 & 25 & 50 & 1 & 5 & 25 & 50 \\
\hline RNN $(1)$ & 0.07 & 0.18 & 0.28 & 0.34 & 0.10 & 0.23 & 0.34 & 0.40 & 0.15 & 0.28 & 0.40 & 0.46 \\
\hline RNN $(2)$ & 0.08 & 0.21 & 0.33 & 0.39 & 0.11 & 0.24 & 0.36 & 0.43 & 0.13 & 0.27 & 0.38 & 0.46 \\
\hline RNN $(3)$ & 0.09 & 0.21 & 0.33 & 0.39 & 0.12 & 0.25 & 0.36 & 0.43 & 0.13 & 0.26 & 0.39 & 0.45 \\
\hline RNN $(4)$ & 0.05 & 0.15 & 0.26 & 0.31 & 0.08 & 0.18 & 0.28 & 0.35 & 0.09 & 0.20 & 0.32 & 0.40 \\
\hline RNN $(5)$ & 0.06 & 0.15 & 0.24 & 0.29 & 0.09 & 0.19 & 0.28 & 0.33 & 0.10 & 0.20 & 0.31 & 0.37 \\
\hline \hline RNN $(\tau)$ & 0.07 & 0.11 & 0.11 & 0.12 & 0.07 & 0.12 & 0.15 & 0.14 & 0.15 & 0.19 & 0.16 & 0.16 \\
\hline \hline AWD $(1)$ & 0.09 & 0.21 & 0.30 & 0.35 & 0.11 & 0.22 & 0.32 & 0.38 & 0.15 & 0.27 & 0.37 & 0.43 \\
\hline AWD $(2)$ & 0.09 & 0.22 & 0.33 & 0.40 & 0.12 & 0.23 & 0.35 & 0.41 & 0.13 & 0.25 & 0.36 & 0.42 \\
\hline AWD $(3)$ & 0.10 & 0.22 & 0.34 & 0.40 & 0.12 & 0.24 & 0.35 & 0.41 & 0.13 & 0.24 & 0.35 & 0.41 \\
\hline AWD $(4)$ & 0.06 & 0.17 & 0.27 & 0.33 & 0.08 & 0.18 & 0.26 & 0.31 & 0.09 & 0.19 & 0.27 & 0.34 \\
\hline AWD $(5)$ & 0.08 & 0.17 & 0.26 & 0.31 & 0.09 & 0.18 & 0.27 & 0.31 & 0.10 & 0.19 & 0.28 & 0.34 \\
\hline \hline AWD $(\tau)$ & 0.06 & 0.12 & 0.10 & 0.11 & 0.10 & 0.12 & 0.14 & 0.16 & 0.15 & 0.17 & 0.19 & 0.18 \\
\hline
\end{tabular}

Table 6: Completion rates broken down per readability level for gap filling systems of variable strength tested on the Weebit dataset. Kendall tau-b correlation is reported as $\tau$.

from automatic scraping (e.g. "Your web browser does not have JavaScript switched on at the moment."). Empty and duplicate documents were removed (around 400 texts), moreover, we used spacy-cld ${ }^{10}$ (which uses CLD2 from Google) to detect the main language of a document and get rid of documents that were not written in English (around 60 texts in French, German, Spanish and Gaelic). In all our test and evaluation datasets, word tokenization and POS tags inference are performed with SpaCy. ${ }^{11}$ Following this processing and as described in Section 2.3, passages and cloze positions are selected and fixed for all experiments to have a deterministic experimental setup. All the results presented below are computed using 500 randomly chosen texts for each readability level.

Since our objective is to study the correlation between gap filling performance and readability level, we compute two main metrics for each of the experimental conditions below: (a) the precision of comprehension systems broken down by

\footnotetext{
${ }^{10}$ https://github.com/nickdavidhaynes/ spacy-cld

${ }^{11}$ http: / / spacy.io
}

complexity group; (b) the correlation (Kendall's Tau) between complexity classes and completion rates. ${ }^{12}$

\subsection{Results}

Our main results are presented in Table 5, 6 and 7 where we report the completion rates and correlations for all the systems compared in our experiments.

A first observation is that completion rates (especially p@1) are overall distributed as expected across models/training corpora. Training on WikiText-2 yields results that are consistently worse than for the other two corpora; for both RNN implementations, the average success rate for WikiText-2 and WikiText-103 are very comparable. Interestingly, results for the easiest readability levels (Weebit 1 and 2) are higher for models trained on Wiki-Simple than on WikiText-103 and WikiText-2. Regarding models, for WikiText-2 and WikiText-103 the RNN implementation of (Merity et al., 2018)

\footnotetext{
${ }^{12}$ We use the Tau-b statistic which makes adjustments for ties (documents in the same complexity class).
} 
is slightly better than ours, while our model performance is better for Wiki-Simple, which is consistent with the perplexity scores in Table 2

\begin{tabular}{|l||r|r|}
\hline \multirow{2}{*}{\multicolumn{1}{|c||}{ p@ ... }} & \multicolumn{2}{|c|}{ WebText } \\
\cline { 2 - 3 } & 1 & 5 \\
\hline OSE $(1)$ & 0.22 & 0.42 \\
\hline OSE $(2)$ & 0.21 & 0.42 \\
\hline OSE $(3)$ & 0.20 & 0.40 \\
\hline \hline OSE $(\tau)$ & 0.05 & 0.04 \\
\hline \hline Weebit $(1)$ & 0.18 & 0.35 \\
\hline Weebit $(2)$ & 0.18 & 0.37 \\
\hline Weebit $(3)$ & 0.21 & 0.39 \\
\hline Weebit $(4)$ & 0.13 & 0.27 \\
\hline Weebit $(5)$ & 0.13 & 0.29 \\
\hline \hline Weebit $(\tau)$ & 0.13 & 0.14 \\
\hline
\end{tabular}

Table 7: Completion rates broken down per readability level for the GPT-2 gap filling system pre-trained on WebText and tested on the Weebit and OSE datasets. Kendall tau-b correlation is reported as $\tau$.

As expected, GPT-2 scores in Table 7 vastly outperform all RNN-based approaches, with completion rates (p@1 / p@5) that approximately double the completion rates of the other systems.

We can conclude that our set of models is quite diverse, even though we would have expected to get more diversity across training corpora from our RNNs, suggesting that we may have to move away from the Wikipedia domain in future experiments.

If we now look at the variance of completion rates across difficulty levels, the overall picture is less clear. Overall, the most consistent results are obtained with OSE than with Weebit: for the former we almost always see better completion rates for simpler texts, across systems, training corpus and metrics, even though the differences are often small. Results for Weebit are more difficult to analyze: all RNN models seem to achieve their best completion rates for Weebit level 3, which is consistently higher than levels 4 and 5 (which is fine), but also higher than for level 1 and 2 (which is contradictory to our expectations). In fact, these easiest texts seem difficult to predict for all models; as mentioned above, for RNNs, training on the simpler Wiki-Simple gives a slight hedge over the other language models, which we also see as a positive sign. Overall however, the observed correlation scores remain small and insignificant, for all systems and metrics. Our best results are in the region $[0.1,0.2]$ for OSE and Weebit. In comparison, the correlation for Flesh-Kincaid grade level score is 0.48 for OSE and 0.61 for Weebit.

A first conclusion is that with current machine comprehension systems, as implemented in this study, we show gap filling performances that are not significantly impacted by the actual readability level of texts. Therefore, their performance in cloze tests can not reliably be used to infer the difficulty of a text.

\section{Discussion}

\subsection{Cloze tests and morphological tags}

The results in Section 3 were obtained with a naive cloze test generation strategy, where deleted tokens are selected uniformly at random. There are several ways in which this approach can be problematic. Indeed, it is already a well documented fact that RNNs are far better at correctly predicting determiners, prepositions, or even verbs, than at predicting nouns (common or proper) that often require a much larger textual context (Hill et al., 2016).

This is also what we observe in Table 8 where we give the completion rate averaged over all texts in OSE corpora, broken down by part-of-speech (POS),${ }^{13}$ for all our models.

\begin{tabular}{|l||r|r|r|r||}
\hline & verb & noun & punct & det \\
\hline RNN-WT2 $(\lambda)$ & 0.03 & 0.08 & 0.76 & 0.61 \\
RNN-WTS IMP LE $(\lambda)$ & 0.05 & 0.14 & 0.81 & 0.66 \\
RNN-WT103 $(\lambda)$ & 0.04 & 0.11 & 0.82 & 0.59 \\
RNN-WT2 $(\tau)$ & 0.02 & 0.06 & 0 & 0 \\
RNN-WTSIMP LE $(\tau)$ & 0.03 & 0.08 & 0.04 & 0.05 \\
RNN-WT103 $(\tau)$ & 0.01 & 0.03 & 0.01 & 0 \\
\hline \hline AWD-WT2 $(\lambda)$ & 0.02 & 0.08 & 0.81 & 0.61 \\
AWD-WTSIMPLE $(\lambda)$ & 0.05 & 0.1 & 0.81 & 0.66 \\
AWD-WT103 $(\lambda)$ & 0.05 & 0.09 & 0.81 & 0.6 \\
AWD-WT2 $(\tau)$ & -0.01 & 0.06 & 0.02 & 0.01 \\
AWD-WTSIMPLE $(\tau)$ & 0.03 & 0.07 & 0.03 & 0.01 \\
AWD-WT103 $(\tau)$ & -0.03 & 0.06 & 0.02 & -0.03 \\
\hline \hline GPT2 $(\lambda)$ & 0.24 & 0.39 & 0.87 & 0.64 \\
GPT2 $(\tau)$ & 0.04 & 0 & 0.01 & -0.02 \\
\hline
\end{tabular}

Table 8: Completion rates ( $\lambda$ with p@5) broken down per model and POS tag and Kendall-Tau $(\tau)$ correlation between completion rates and their respective level on the OSE dataset.

A first consequence is that easy-to-predict tokens, such as punctuations or determiners, will artificially increase the completion rate, even for texts that should be difficult to complete.

This would not be so much of a problem if the distribution of POS was constant across readability levels, an assumption that is also well known to be unrealistic - in fact, POS ratios have repeatedly

\footnotetext{
${ }^{13}$ Again, we use SpaCy to compute part-of-speech tags.
} 


\begin{tabular}{|c|l|l|}
\hline Level & Title & CR \\
\hline K0 & Goldlilocks and the Three Bears & 0 \\
K1 & The Courage of Sarah Noble & 0 \\
K1 & Flat Stanley & 0 \\
K2 & The Velveteen Rabbit & 0.07 \\
K2 & Ribsy & 0.07 \\
K3 & Stuart Little & 0.20 \\
K3 & Peter Pan & 0.33 \\
K4 & Prince Caspian & 0.07 \\
K4 & Alice's Adventures in Wonderland & 0.07 \\
K5 & Robinson Crusoe & 0.13 \\
K5 & The Voyage of the Dawn Treader & 0.07 \\
K6 & The Red Badge of Courage & 0.13 \\
K6 & The Last Battle & 0.07 \\
K7 & The Count of Monte Cristo & 0.07 \\
K7 & The Black Arrow & 0.27 \\
K8 & Hamlet & 0 \\
K8 & The Sword in the Stone & 0.07 \\
K+ & The Art of War & 0.07 \\
\hline
\end{tabular}

Table 9: A literary test set with completion rates from our RNN implementation trained on WikiText-2 using p@ 1 and 5 chunks of text per document.

been found to yield useful features when predicting readability (e.g. François and Fairon (2012)). The main consequence for our argument is that randomly selecting gaps will yield uneven POS distribution across readability levels, therefore biaising the overall result.

As suggested by the variance of the results in Table 8, this confounding factor cannot be ignored. Part-of-speech tags with the best rank correlation between their completion rate and their text's level are nouns and verbs; their occurrences are however lesser than punctuations and determiners, which means that an uniformly random gap selection strategy is biased by morphological tags that are worse predictors of text readability for our models.

\subsection{Experiment with literary texts}

The test datasets used in this study are quite similar in content, and all correspond to news articles intended for readers with variable levels of proficiency. In this subsection, we run a small confirmation study with an alternative test set based on literary texts. We use extracts from classical fictional books (see list in Table 9 where we also report the associated readability level and completion rate).

Kendall rank coefficient between these rates and their respective levels is equal to -0.28 .

Here again, levels are not distinguishable based on the observed completion rates. However, as seen above in section $\S 3.3$ our systems perform better when we release the exact match constraint for the precision@N metrics. We expect to increase the Kendall rank metric on larger experiments with this dataset in our future work.

\section{Conclusion and outlook}

In this paper, we have ran a preliminary study regarding the ability of basic reading comprehension systems, here implemented as mere neural language models, to recognize variance in the difficulty of their input texts. Using randomly uniformly generated cloze tests to measure comprehension, we found that completion rates seem to deliver a small, yet insufficient, signal regarding the readability level of a text. Our strongest comprehension system, based on the recent GPT2 model, is the worst of all, and does not help to distinguish between simple and more complex texts. Complementary experiments show that better controlling for POS distribution across corpora is likely to improve, albeit by a small margin our results: and that testing with other genre of texts might yield similar conclusions.

This pilot study only scratches at the surface of the problem, and we intend to continue this research in several directions. First, we need to continue the exploration of comprehension systems of controllable strength, using either more sophisticated language modelling architectures, or even considering full-fledge machine comprehension systems. A second line of study will consider alternative procedures for generating and filling cloze tests: generation could for instance restrict to specific gap types, based on their morphosyntactic properties or blank word parts instead of complete words; filling could for instance increase the context rightwards. Finally, we feel that it will also be crucial to make more direct experiments of the ability of automatic system to consistently reproduce the performance of human subjects, notably in the context of the evaluation of educational material, and we aim to develop experimental protocols involving controlled populations of language learners.

\section{References}

Rami Al-Rfou, Dokook Choe, Noah Constant, Mandy Guo, and Llion Jones. 2018. Character-level language modeling with deeper self-attention. In Proceedings of the Thirty-Third AAAI Conference on Artificial Intelligence, AAAI, Honolulu, Hawaï.

Frédéric Bimbot, Marc El-Bèze, Stéphane Igounet, 
Michèle Jardino, Kamel Smaili, and Imed Zitouni. 2001. An alternative scheme for perplexity estimation and its assessment for the evaluation of language models. Computer Speech \& Language, 15(1):1-13.

Dominique Brunato, Lorenzo De Mattei, Felice Dell'Orletta, Benedetta Iavarone, and Giulia Venturi. 2018. Is this sentence difficult? do you agree? In Proceedings of the 2018 Conference on Empirical Methods in Natural Language Processing, pages 2690-2699, Brussels, Belgium.

Stanley F Chen and Joshua Goodman. 1999. An empirical study of smoothing techniques for language modeling. Computer Speech \& Language, 13(4):359-394.

Kevyn Collins-Thompson. 2014. Computational assessment of text readability: A survey of current and future research. ITL-International Journal of Applied Linguistics, 165(2):97-135.

Kevyn Collins-Thompson and James P. Callan. 2004. A language modeling approach to predicting reading difficulty. In HLT-NAACL 2004: Main Proceedings, pages 193-200, Boston, Massachusetts, USA.

Bhuwan Dhingra, Hanxiao Liu, Zhilin Yang, William Cohen, and Ruslan Salakhutdinov. 2017. Gatedattention readers for text comprehension. In Proceedings of the 55th Annual Meeting of the Association for Computational Linguistics (Volume 1: Long Papers), pages 1832-1846, Vancouver, Canada.

Arne Dietrich. 2004. Neurocognitive mechanisms underlying the experience of flow. Consciousness and Cognition, 13(4):746 - 761 .

William H DuBay. 2007. Smart language. Readers, Readability, and the Grading of Text. Costa Mesa: Impact Information.

Jeffrey L. Elman. 1990. Finding structure in time. Cognitive Science, 14(2):179-211.

Lijun Feng, Noémie Elhadad, and Matt Huenerfauth. 2009. Cognitively motivated features for readability assessment. In Proceedings of the 12th Conference of the European Chapter of the ACL, EACL 2009, pages 229-237, Athens, Greece.

Thomas François and Cédrick Fairon. 2012. An AI readability formula for French as a foreign language. In Proceedings of the 2012 Joint Conference on Empirical Methods in Natural Language Processing and Computational Natural Language Learning, pages 466-477.

Karl Moritz Hermann, Tomas Kocisky, Edward Grefenstette, Lasse Espeholt, Will Kay, Mustafa Suleyman, and Phil Blunsom. 2015. Teaching machines to read and comprehend. In Advances in Neural Information Processing Systems 28, pages 1693 1701. Curran Associates, Inc.
Felix Hill, Antoine Bordes, Sumit Chopra, and Jason Weston. 2016. The Goldilocks principle: reading children's books with explicit memory representations. In Proceedings of the International Conference on Learning Representations, ICLR, San Juan, Puerto Rico.

Sepp Hochreiter and Jürgen Schmidhuber. 1997. Long short-term memory. Neural computation, 9(8):1735-1780.

Hakan Inan, Khashayar Khosravi, and Richard Socher. 2017. Tying word vectors and word classifiers: A loss framework for language modeling. In Proceedings of the International Conference on Representation Learning, ICLR, Toulon, France.

Robin Jia and Percy Liang. 2017. Adversarial examples for evaluating reading comprehension systems. In Proceedings of the 2017 Conference on Empirical Methods in Natural Language Processing, pages 2021-2031.

Divyansh Kaushik and Zachary C. Lipton. 2018. How much reading does reading comprehension require? a critical investigation of popular benchmarks. In Proceedings of the 2018 Conference on Empirical Methods in Natural Language Processing, pages 5010-5015, Brussels, Belgium.

Yoon Kim, Yacine Jernite, David A Sontag, and Alexander M. Rush. 2016. Character-aware neural language models. In Proceedings of the Annual Meeting of the American Association for Artificial Intelligence, AAAI 16.

J Peter Kincaid, Robert P Fishburne Jr, Richard L Rogers, and Brad S Chissom. 1975. Derivation of new readability formulas (automated readability index, fog count and Flesch reading ease formula) for navy enlisted personnel. Technical report, Institute for Simulation and Training, University of Central Florida.

Peter J. Liu, Mohammad Saleh, Etienne Pot, Ben Goodrich, Ryan Sepassi, Lukasz Kaiser, and Noam Shazeer. 2018. Generating wikipedia by summarizing long sequences. In Proceedings of the International Conference on Learning Representations, ICLR, Vancouver, BC, Canada.

Gábor Melis, Chris Dyer, and Phil Blunsom. 2018. On the state of the art of evaluation in neural language models. In Proceedings of the International Conference on Learning Representations, ICLR, Vancouver, BC, Canada.

Stephen Merity, Nitish Shirish Keskar, and Richard Socher. 2018. Regularizing and optimizing LSTM language models. In Proceedings of the International Conference on Learning Representations, ICLR.

Stephen Merity, Caiming Xiong, James Bradbury, and Richard Socher. 2017. Pointer sentinel mixture 
models. In Proceedings of the International Conference on Learning Representations, ICLR, Toulon, France.

Tomáš Mikolov, Martin Karafiát, Lukáš Burget, Jan Černockỳ, and Sanjeev Khudanpur. 2010. Recurrent neural network based language model. In Eleventh Annual Conference of the International Speech Communication Association (InterSpeech), pages 1045-1048, Makuhari, Chiba, Japan.

John W. Oller Jr. 1973. Cloze tests of second language proficiency and what they measure. Language Learning, 23(1):105-118.

Denis Paperno, Germán Kruszewski, Angeliki Lazaridou, Ngoc Quan Pham, Raffaella Bernardi, Sandro Pezzelle, Marco Baroni, Gemma Boleda, and Raquel Fernandez. 2016. The LAMBADA dataset: Word prediction requiring a broad discourse context. In Proceedings of the 54th Annual Meeting of the Association for Computational Linguistics (Volume 1: Long Papers), pages 1525-1534, Berlin, Germany.

Sarah E. Petersen and Mari Ostendorf. 2009. A machine learning approach to reading level assessment. Computer Speech \& Language, 23(1):89 - 106.

Ofir Press and Lior Wolf. 2017. Using the output embedding to improve language models. In Proceedings of the 15th Conference of the European Chapter of the Association for Computational Linguistics: Volume 2, Short Papers, pages 157-163, Valencia, Spain.

Alec Radford, Jeffrey Wu, Rewon Child, David Luan, Dario Amodei, and Ilya Sutskever. 2019. Language models are unsupervised multitask learners. Technical report, OpenAI.

Pranav Rajpurkar, Jian Zhang, Konstantin Lopyrev, and Percy Liang. 2016. Squad: 100,000+ questions for machine comprehension of text. In Proceedings of the 2016 Conference on Empirical Methods in Natural Language Processing, pages 2383-2392, Austin, Texas.

Matthew Richardson, Christopher J.C. Burges, and Erin Renshaw. 2013. MCTest: A challenge dataset for the open-domain machine comprehension of text. In Proceedings of the 2013 Conference on Empirical Methods in Natural Language Processing, pages 193-203, Seattle, Washington, USA.

Herbert Robbins and Sutton Monro. 1951. A stochastic approximation method. The Annals of Mathematical Statistics, 22(3):400-407.

Sarah Schwarm and Mari Ostendorf. 2005. Reading level assessment using support vector machines and statistical language models. In Proceedings of the 43rd Annual Meeting of the Association for Computational Linguistics (ACL'05), pages 523-530, Ann Arbor, Michigan.
Rico Sennrich, Barry Haddow, and Alexandra Birch. 2016. Neural machine translation of rare words with subword units. In Proceedings of the 54th Annual Meeting of the Association for Computational Linguistics (Volume 1: Long Papers), pages 17151725 , Berlin, Germany.

Ilya Sutskever, James Martens, and Geoffrey Hinton. 2011. Generating text with recurrent neural networks. In Proceedings of the 28th International Conference on International Conference on Machine Learning, ICML'11, pages 1017-1024.

Wilson L. Taylor. 1953. Cloze procedure: A new tool for measuring readability. Journalism Quarterly, 30:415-433.

Sowmya Vajjala and Ivana Lucic. 2018. Onestopenglish corpus: A new corpus for automatic readability assessment and text simplification. In Proceedings of the Thirteenth Workshop on Innovative Use of NLP for Building Educational Applications, pages 297-304, New Orleans, Louisiana.

Sowmya Vajjala and Detmar Meurers. 2012. On improving the accuracy of readability classification using insights from second language acquisition. In Proceedings of the seventh workshop on building educational applications using NLP, pages 163-173.

Sowmya Vajjala and Detmar Meurers. 2014. Exploring measures of "readability" for spoken language: Analyzing linguistic features of subtitles to identify age-specific tv programs. In Proceedings of the $3 r d$ Workshop on Predicting and Improving Text Readability for Target Reader Populations (PITR), pages 21-29.

Ashish Vaswani, Noam Shazeer, Niki Parmar, Jakob Uszkoreit, Llion Jones, Aidan N Gomez, Łukasz Kaiser, and Illia Polosukhin. 2017. Attention is all you need. In Advances in Neural Information Processing Systems, pages 5998-6008.

Qizhe Xie, Guokun Lai, Zihang Dai, and Eduard Hovy. 2018. Large-scale Cloze test dataset created by teachers. In Proceedings of the 2018 Conference on Empirical Methods in Natural Language Processing, pages 2344-2356, Brussels, Belgium.

Zhilin Yang, Zihang Dai, Ruslan Salakhutdinov, and William W. Cohen. 2018. Breaking the softmax bottleneck: A high-rank RNN language model. In Proceedings of the International Conference on Learning Representations, ICLR.

Adams Wei Yu, David Dohan, Minh-Thang Luong, Rui Zhao, Kai Chen, Mohammad Norouzi, and Quoc V. Le. 2018. QANet: Combining local convolution with global self-attention for reading comprehension. In Proceedings of the International Conference on Learning Representations, ICLR.

Geoffrey Zweig and Chris J.C. Burges. 2012. A challenge set for advancing language modeling. In Proceedings of the NAACL-HLT 2012 Workshop: Will 
We Ever Really Replace the N-gram Model? On the Future of Language Modeling for HLT, pages 2936, Montréal, Canada. 